\title{
Paper Case Report Form
}

National Cancer Institute

\section{Source}

National Cancer Institute. Paper Case Report Form. NCI Thesaurus. Code C142625.

A case report form that is printed on paper. 